\title{
Using the Grey-TOPSIS Method to Assess the Functioning of the Occupational Risk Management
}

\author{
Joanna Tabor ${ }^{1, *}$ \\ ${ }^{1}$ Czestochowa University of Technology, Faculty of Management, Częstochowa 42 - \\ 201, ul. J.H. Dąbrowskiego 69, Poland
}

\begin{abstract}
The objective of this paper is to analyse the possibility to use the Grey-TOPSIS method to assess the occupational risk management $(\mathrm{ORM})$, in the situation whereby linguistic variables were used in the assessment process. The TOPSIS method has already been in use for a number of years to assess alternative solutions in many areas other than the work safety area. This paper remedies some shortcomings in using the Grey Systems Theory (GST) in ORM, and constitutes an original application of Grey-TOPSIS in the work safety area. Considering that assessment of the ORM process involves estimation of performance particular components of this process, the eight sub-processes were adopted for assessment purposes. The degree of fulfilment of a given subprocesses was assigned a respective verbal grade from Completely Wrong to Perfect. In performing this assessment, systems being in place in four furniture-manufacturing companies were selected to minimise impacts of external factors upon the ORM in these companies. By using the GreyTOPSIS method, it was possible to rank these companies from the point of view of functioning of the ORM process, and to identify the best and the weakest company in this respect. Further research work should be conducted with the aim to develop some new hybrid, multi-criterion assessment methods, which will develop within the frameworks of the GST.
\end{abstract}

\section{Introduction}

Occupational risk management is the key process in the area of providing for safe and healthy working conditions in all types of organizations, regardless of how big they are. The need to perform this process results from many international and local statutory regulations, whereas plentiful risk analysis / assessment models, which are available in the literature of the subject [1 -5], confirm the need to improve work health and safety management quality. Assessment of the performance level of occupational risk management processes is especially important in situations, whereby both management systems used to handle risks that come from various sources [6] and management systems

\footnotetext{
${ }^{*}$ Corresponding author: joanna.tabor@,wz.pcz.pl
} 
used to handle various operational areas of the organization must be integrated [7]. Broadly speaking, it can be said that occupational risk management is a continuous and complex process of assessing and eliminating or restricting risks [4, 8]. High complexity of this process results in high number of criteria of assessment of the way it works, which can be different, both quantitative and qualitative, in nature. Furthermore, within the frameworks of the process itself, these criteria can also have different levels of significance, manifesting themselves in their varying influence upon the end result, i.e. the overall assessment of how the process works. All of this suggests that the problem of assessment of how the occupational risk management process works should be treated as a multi-criteria decisionmaking problem, and that appropriate decision-making tools, which have proven themselves well in other areas, should be used for such assessment purposes. Although decision-making processes constitute integral part of work health and safety management systems, the use of multi-criteria decision-making tools in the occupational risk management area is still rarely practiced, and poorly described in the literature of the subject. At the same time, due to the fact that human thinking is highly complex and unclear in nature, it is better in many cases to use qualitative information, using the linguistic approach, rather than quantitative information which may require engagement of substantial resources to be collected. It is especially important in many practical situations regarding decision-making and assessment processes [9]. In the linguistic approach, natural language words (such as "wrong", "good", "low", or "high") are treated as variables that are processed according to some determined procedures [10 - 13].

In view of the above, the objective of this paper is to review the possibility to use the grey TOPSIS method (Technique for Order Preference by Similarity to Ideal Solution) to assess occupational risk management processes in selected companies, in the situation when linguistic variables are used for assessment purposes.

\section{Occupational risk management as a multi-criteria problem}

In line with the basic risk management concept as such, the occupational risk management process, in principle, consists of two main parts: risk analysis / assessment, and risk control $[2,8]$. In the risk analysis / assessment area, the following four basic actions are performed: collecting information required for risk assessment purposes, identification of hazards, risk estimation, and setting the risk acceptability level. In the risk control area, the following five basic actions are carried out: analysis of preventive measures implementation options, adopting decisions on proper measures that should be selected, implementation of these measures, monitoring and assessment of their efficiency. Some determined problems, which must be faced in the process of implementation of the particular measures, are associated with each of these measures or sub-processes.

At the risk analysis stage, the key problem is to properly select sources of information in hazard identification processes, and to use the right data collection methods, as the same hazard may provoke different consequences [14]. One of the problems at the risk estimation stage is to properly select methods in the context of the identified hazards. There are many risk estimation methods $[2,15]$, but not all of them are appropriate for a given situation. At the risk assessment stage, the key problem is to properly set the risk tolerance level, the risk control level, and risk non-acceptance level, in the situation when decision in this regard depends on different (financial, human resources, organizational, and other) conditions [16]. The key problem at the preventive options analysis stage is to adopt the appropriate methodology to select solutions which should be considered from the standpoint of such factors as: efficiency of the solution, its cost effectiveness, feasibility and completion time, its psychological effect, and its subjective reception by the employees involved, etc. [17]. Decisions pertaining to preventive measures involve problems connected with the proper 
planning of actions, allocation of resources, appointing persons responsible, and setting action completion times according to the organization's capacity at a given time [3]. The preventive measures implementation stage covers problems related to practical completion of any planned actions in line with the adopted schedule [1], while the monitoring stage involves problems connected with proper selection of the scope of actions and the level of rigorousness of day-to-day supervision of the planned actions [2]. Problems encountered at the last stage in the risk management cycle, i.e. at the preventive measures efficiency evaluation stage, pertain to proper assessment of the actual risk level reduction after selected preventive measures have been applied.

Due to all these problems as described above, a decision maker may fail to achieve the objective that the occupational risk management process should address, which is the ongoing and genuine improvement of work conditions.

\section{TOPSIS method and grey numbers}

Due to the complex nature of the occupational risk management process, we can treat the issues of comparison and assessment of the level of risk management process performance as decision-making issues that involve many criteria and a finite number of possible decision-making variants; and try to solve them by using a method or tool from the multicriteria decision-making area. The literature of the subject offers plentiful examples of such methods or tools (e.g. AHP, TOPSIS, VIKOR, PROMETHEE), with varying degree of complexity and capacity to solve determined problems [18]. One of the best known methods in this respect is the TOPSIS method, which is used in decision-making processes in linear streamlining of variants [19].This method is based upon using the measure of relative distance from the best solution, which constitutes the pattern, and from the worst method, which constitutes the anti-pattern.

The basic objective of the TOPSIS method is to identify the solution (variant), which would feature the maximum relative proximity to the pattern and the minimum relative proximity to the anti-pattern. The TOPSIS method is constantly evolving as new methodologies arise. Currently, in practice, the so-called fuzzy TOPSIS method is most frequently applied [20], which makes use of the fuzzy sets theory. On the other hand, it is proposed in this work to use the grey systems theory, and to apply the grey TOPSIS method. The grey systems theory (GST) is the latest methodology used to analyze and assess systems in the situation whereby information upon these systems is uncertain and incomplete. The grey systems theory makes it possible to bypass necessary assumptions concerning statistical, rough or fuzzy methods, and results obtained by using grey numbers, are more accurate than in any other approach [21]. Let $X$ be the universal set. Then a grey set $G$ of $X$ is defined by two mappings $\bar{\mu}_{G}(X)$ and $\underline{\mu}_{G}(X): \bar{\mu}_{G}(X): X \rightarrow[0,1]$ and $\underline{\mu}_{G}(X): X \rightarrow[0,1]$ such that $\bar{\mu}_{G}(X) \geq \underline{\mu}_{G}(X), x \in X$. Since the lower limit $\otimes G=[\underline{G}, \infty)$ and upper limit $\otimes G=(-\infty, \bar{G}$ can be estimated, $\mathrm{G}$ is defined as interval grey number $\otimes G=[\underline{G}, \bar{G}][21]$. The basic operations on grey numbers are performed in the following manner:

$$
\begin{aligned}
& \otimes G_{1}+\otimes G_{2}=\left[\underline{G}_{1}+\underline{G}_{2}, \bar{G}_{1}+\bar{G}_{2}\right] ; \otimes G_{1}-\otimes G_{2}=\left[\underline{G_{1}}-\bar{G}_{2}, \bar{G}_{1}-\underline{G}_{2}\right] \\
& \otimes G_{1} \times \otimes G_{2}=\left[\min \left(\underline{G}_{1} \underline{G_{2}}, \underline{G}_{1} \bar{G}_{2}, \bar{G}_{1} \underline{G}_{2}, \bar{G}_{1} \bar{G}_{2}\right), \max \left(\underline{G_{1}} \underline{G}_{2}, \underline{G}_{1} \bar{G}_{2}, \bar{G}_{1} \underline{G_{2}}, \bar{G}_{1} \bar{G}_{2}\right)\right] \\
& \otimes G_{1} \div \otimes G_{2}=\left[\underline{G_{1}}, \bar{G}_{1}\right] \times\left[\frac{1}{\underline{G}_{2}}, \frac{1}{\bar{G}_{2}}\right]
\end{aligned}
$$

At the moment, the grey systems theory is used in many disciplines of engineering, medical, and social sciences, as evidenced by the systematically growing number of publications concerning its practical applications, including in broadly-comprehended 
safety engineering and in management of economic systems of different sizes. One of the fastest-developing applications is use of the GST theory in classical and modern multicriteria decision-making tools [21], and the use of grey numbers in the classical version of the TOPSIS method is a good example of such an application.

\section{Research methodology}

The objective of this research work was to analyze a possibility to use the grey TOPSIS method to assess occupational risk management processes in selected companies, in the situation when linguistic variables were used to assess processes. The alternatives ranking procedure we have worked out consists of the following stages:

1) Assess the decision-making criteria using linguistic variables,

2) Assess the significance level of the decision-making criteria, and aggregate such assessments using the arithmetic mean method:

$$
\otimes W_{j}=\frac{1}{K}\left[\otimes W_{j}^{1}+\otimes W_{j}^{2}+\cdots+\otimes W_{j}^{K}\right] \text { where }: \otimes W_{j}^{K}=\left[\underline{W}_{j}^{K}, \bar{W}_{j}^{K}\right]
$$

3) Assess the alternatives using linguistic variables, and aggregate such assessments using the arithmetic mean method:

$$
\otimes G_{i j}=\frac{1}{K}\left[\otimes G_{i j}^{1}+\otimes G_{i j}^{2}+\cdots+\otimes G_{i j}^{K}\right]
$$

where: $\otimes G_{i j}^{K},(i=1,2, \ldots, m ; j=1,2, \ldots, n)$ is an assessment of the criterion by the $\mathrm{k}^{\text {th }}$ decision-maker, which is represented by a grey number in a form: $\otimes G_{i j}^{K}=\left[\underline{G}_{i j}^{K}, \bar{G}_{i j}^{K}\right]$.

4) Build the grey decision-making matrix in the following form:

$$
D=\left[\begin{array}{cccc}
\otimes G_{11} & \bigotimes G_{12} & \cdots & \bigotimes G_{1 n} \\
\otimes G_{21} & \bigotimes G_{22} & & \otimes G_{2 n} \\
\vdots & \vdots & \ddots & \vdots \\
\otimes G_{m 1} & \bigotimes G_{m 2} & \cdots & \otimes G_{m n}
\end{array}\right]
$$

5) Build the normalized grey decision-making matrix in the following form:

$$
\begin{aligned}
& D^{*}=\left[\begin{array}{cccc}
\otimes G_{11}^{*} & \bigotimes G_{12}^{*} & \cdots & \otimes G_{1 n}^{*} \\
\otimes G_{21}^{*} & \bigotimes G_{22}^{*} & & \otimes G_{2 n}^{*} \\
\vdots & \vdots & \ddots & \vdots \\
\otimes G_{m 1}^{*} & \bigotimes G_{m 2}^{*} & \cdots & \otimes G_{m n}^{*}
\end{array}\right], \text { where } \\
& \otimes G_{i j}^{*}=\left[\frac{\underline{G}_{i j}}{G_{j}^{\text {max }}}, \frac{\bar{G}_{i j}}{G_{j}^{\text {max }}}\right] \text { and } G_{j}^{\max }=\max _{1 \leq i \leq m}\left\{\bar{G}_{i j}\right\}
\end{aligned}
$$

6) Build the weighted normalized grey decision-making matrix in the following form:

$$
D_{W}^{*}=\left[\begin{array}{cccc}
\otimes V_{11} & \otimes V_{12} & \cdots & \otimes V_{1 n} \\
\otimes V_{21} & \otimes V_{22} & & \otimes V_{2 n} \\
\vdots & \vdots & \ddots & \vdots \\
\otimes V_{m 1} & \otimes V_{m 2} & \cdots & \otimes V_{m n}
\end{array}\right] \text {, where }: \otimes V_{i j}=\otimes G_{i j}^{*} \times \otimes W_{j}
$$

7) Identify the pattern in line with the following assumption that for the set $m$ of possible alternatives $A=\left\{A_{1}, A_{2}, A_{3}, \ldots A_{m}\right\}$, pattern $A^{\max }$ is to be determined as follows:

$$
A^{\max }=\left\{\otimes V_{1}^{\max }, \otimes V_{2}^{\max }, \ldots, \otimes V_{n}^{\max }\right\}
$$

where $A^{\max }=\left\{\left[\max _{1 \leq i \leq m} \underline{V}_{i 1}, \max _{1 \leq i \leq m} \bar{V}_{i 1}\right],\left[\max _{1 \leq i \leq m} V_{i 2}, \max _{1 \leq i \leq m} \bar{V}_{i 2}\right], \ldots,\left[\max _{1 \leq i \leq m} \underline{V}_{i n}, \max _{1 \leq i \leq m} \bar{V}_{i n}\right]\right\}$ 8) Identify the anti-pattern in line with the following assumption that for the set $m$ of possible alternatives $\mathrm{A}=\left\{\mathrm{A}_{1}, \mathrm{~A}_{2}, \mathrm{~A}_{3}, \ldots \mathrm{A}_{m}\right\}$, anti-pattern $\mathrm{A}^{\text {min }}$ is to be determined as follows:

$$
A^{\min }=\left\{\otimes V_{1}^{\min }, \otimes V_{2}^{\min }, \ldots, \otimes V_{n}^{\min }\right\}
$$


where $A^{\min }=\left\{\left[\min _{1 \leq i \leq m} V_{i 1}, \min _{1 \leq i \leq m} \bar{V}_{i 1}\right],\left[\min _{1 \leq i \leq m} V_{i 2}, \min _{1 \leq i \leq m} \bar{V}_{i 2}\right], \ldots,\left[\min _{1 \leq i \leq m} V_{i n}, \min _{1 \leq i \leq m} \bar{V}_{i n}\right]\right\}$ 9) Calculate distances between the alternatives being compared and pattern $A^{\max }$ and antipattern $A^{\text {min }}$, using formulas:

$$
\begin{gathered}
d_{i}^{+}=\sum_{j=1}^{n} d\left(V_{i j}, V_{j}^{\text {max }}\right) \text {, and } d_{i}^{-}=\sum_{j=1}^{n} d\left(V_{i j}, V_{j}^{\text {min }}\right) \text { for } i=1,2, \ldots m \\
\text { where: } d\left(\otimes V_{A}, \otimes V_{B}\right)=\sqrt{1 / 2\left[\left(V_{A}-\underline{V}_{B}\right)^{2}+\left(\bar{V}_{A}-\bar{V}_{B}\right)^{2}\right]},
\end{gathered}
$$

(10) Establish a synthetic assessment measure for variants $C C_{i}$ using relative proximity of variant assessments to the pattern and to the anti-pattern:

$$
C C_{i}=\frac{d_{i}^{-}}{d_{i}^{+}+d_{i}^{-}}, i=1,2, \ldots m
$$

The lower the distance of the assessment of the variant away from the pattern, and, at the same time, the higher the distance away from anti-pattern, the closer the value of the measure is to 1 .

(11) Create a ranking for $m$ alternatives based upon linear streamlining synthetic assessment measures in descending order

Trying to structure the problem, we determined criteria $\mathrm{C}$ of assessment of performance level of the occupational risk management process, which corresponded to the following stages of this process: $\mathrm{C}_{1}-$ Identification of hazards, $\mathrm{C}_{2}-$ Risk estimation, $\mathrm{C}_{3}-$ Risk assessment, $\mathrm{C}_{4}-$ Analysis of preventive options, $\mathrm{C}_{5}$-Adopting decisions on preventive measures, $\mathrm{C}_{6}-$ Implementation of preventive measures, $\mathrm{C}_{7}-$ Monitoring preventive measures, $\mathrm{C}_{8}-$ Evaluation of efficiency of preventive measures. Upon having determined our assessment criteria, we identified possible alternative solutions $\mathrm{A}$, based upon four furniture manufacturing companies: $\mathrm{A}_{1}, \mathrm{~A}_{2}, \mathrm{~A}_{3}$, and $\mathrm{A}_{4}$, with a similar portfolio of products, position on the market, and employment level ranging from 50 to 99 persons.

At the problem modelling stage, our four experts $E_{1}-E_{4}$, using an especially prepared questionnaire, first assessed significance levels of criteria $\mathrm{C}_{1}-\mathrm{C}_{8}$, on a seven-degree scale from "insignificant" to "very significant". Then, using the second questionnaire, our experts assessed the performance level of each of the sub-processes in each of the examined companies separately. Each sub-process (or criterion) performance level was assessed regardless of assessment results of the remaining criteria, using the seven-degree scale from "completely wrong" to "perfect".

Significance of this research work results from the fact that in the occupational health and safety area, including occupational risk management processes, there are not enough practically verified tools which could be used by decision makers in their decisions concerning providing for safe and healthy working conditions, especially in the situation when such assessments are made verbally. Therefore, it is justified to take such actions and to conduct such research works, which could change this state of things. Furthermore, intercorporate comparisons and assessments of actions performed as part of occupational risk management processes may constitute a significant source of information for decision makers on the things that should be improved in the processes they run in their companies [22].

\section{Results}

The companies we examined constituted 4 possible alternatives, from $\mathrm{A}_{1}$ to $\mathrm{A}_{4}$, whereas sub-processes constituted the eight criteria we adopted, from $C_{1}$ to $C_{8}$. Verbal assessments of criteria significance were assigned their corresponding grey numbers with the following 
values: insignificant $[0.0,0.1]$, low $[0.1,0.3]$, medium-low $[0.3,0.4]$, medium $[0.4,0.5]$, medium-significant $[0.5,0.6]$, significant $[0.6,0.9]$ and very significant $[0.9,1.0]$.

Table 1 lists grey assessments of significance of the particular criteria or sub-processes.

Table 1. List of grey assessments of sub-process significance levels.

\begin{tabular}{|l|l|l|l|l|l|l|l|l|}
\hline \multirow{2}{*}{$\mathrm{E}$} & \multicolumn{7}{|c|}{ Grey assessments of sub-process significance levels } \\
\cline { 2 - 9 } & $\mathrm{C}_{1}$ & $\mathrm{C}_{2}$ & $\mathrm{C}_{3}$ & $\mathrm{C}_{4}$ & $\mathrm{C}_{5}$ & $\mathrm{C}_{6}$ & $\mathrm{C}_{7}$ & $\mathrm{C}_{8}$ \\
\hline $\mathrm{E}_{1}$ & {$[0.9,1.0]$} & {$[0.6,0.9]$} & {$[0.5,0.6]$} & {$[0.3,0.4]$} & {$[0.6,0.9]$} & {$[0.5,0.6]$} & {$[0.5,0.6]$} & {$[0.3,0.4]$} \\
\hline $\mathrm{E}_{2}$ & {$[0.5,0.6]$} & {$[0.5,0.6]$} & {$[0.4,0.5]$} & {$[0.3,0.4]$} & {$[0.6,0.9]$} & {$[0.5,0.6]$} & {$[0.3,0.4]$} & {$[0.3,0.4]$} \\
\hline $\mathrm{E}_{3}$ & {$[0.6,0.9]$} & {$[0.5,0.6]$} & {$[0.5,0.6]$} & {$[0.4,0.5]$} & {$[0.9,1.0]$} & {$[0.4,0.5]$} & {$[0.3,0.4]$} & {$[0.5,0.6]$} \\
\hline $\mathrm{E}_{4}$ & {$[0.9,1.0]$} & {$[0.6,0.9]$} & {$[0.4,0.5]$} & {$[0.4,0.5]$} & {$[0.6,0.9]$} & {$[0.4,0.5]$} & {$[0.4,0.5]$} & {$[0.6,0.9]$} \\
\hline
\end{tabular}

Using formula (1), we aggregated grey assessments of significance of decision-making criteria, and obtained weights of the particular criteria: $w=\{[0.72,0.88][0.55,0.75]$ $[0.45,0.55][0.35,0.45][0.68,0.92][0.45,0.55][0.38,0.45][0.43,0.58]\}$.

On the other hand, verbal assessments of sub-process performance levels, as part of the particular alternatives, were assigned the following grey numbers: completely wrong $[0.0$, $1.0]$, very poor $[1.0,3.0]$, poor $[3.0,4.0]$, medium $[4.0,5.0]$, good $[5.0,6.0]$, very good $[6.0$, $9.0]$, and perfect $[9.0,10.0]$. Table 2 lists grey assessments of sub-process performance levels in the particular companies.

Table 2. List of grey assessments of sub-process performance levels.

\begin{tabular}{|c|c|c|c|c|c|c|c|c|}
\hline & \multicolumn{8}{|c|}{ Grey assessments of sub-process performance levels } \\
\hline & $\mathrm{C}_{1}$ & $\mathrm{C}_{2}$ & $\mathrm{C}_{3}$ & $\mathrm{C}_{4}$ & $\mathrm{C}_{5}$ & $\mathrm{C}_{6}$ & $\mathrm{C}_{7}$ & $\mathrm{C}_{8}$ \\
\hline \multicolumn{9}{|l|}{$\mathrm{A}_{1}$} \\
\hline $\mathrm{E}_{1}$ & {$[1.0,3.0]$} & {$[5.0,6.0]$} & {$[4.0,5.0]$} & {$[4.0,5.0]$} & {$[3.0,4.0]$} & {$[3.0,4.0]$} & {$[3.0,4.0]$} & {$[3.0,4.0]$} \\
\hline $\mathrm{E}_{2}$ & {$[0.0,1.0]$} & {$[5.0,6.0]$} & {$[3.0,4.0]$} & {$[5.0,6.0]$} & {$[4.0,5.0]$} & {$[4.0,5.0]$} & {$[1.0,3.0]$} & {$[3.0,4.0]$} \\
\hline $\mathrm{E}_{3}$ & {$[1.0,3.0]$} & {$[6.0,9.0]$} & {$[4.0,5.0]$} & {$[4.0,5.0]$} & {$[1.0,3.0]$} & {$[1.0,3.0]$} & {$[4.0,5.0]$} & {$[3.0,4.0]$} \\
\hline $\mathrm{E}_{4}$ & {$[3.0,4.0]$} & {$[4.0,5.0]$} & {$[5.0,6.0]$} & {$[3.0,4.0]$} & {$[3.0,4.0]$} & {$[3.0,4.0]$} & {$[3.0,4.0]$} & {$[3.0,4.0]$} \\
\hline \multicolumn{9}{|l|}{$A_{2}$} \\
\hline $\mathrm{E}_{1}$ & {$[3.0,4.0]$} & {$[1.0,3.0]$} & {$[5.0,6.0]$} & {$[3.0,4.0]$} & {$[5.0,6.0]$} & {$[5.0,6.0]$} & {$[4.0,5.0]$} & {$[3.0,4.0]$} \\
\hline $\mathrm{E}_{2}$ & {$[3.0,4.0]$} & {$[1.0,3.0]$} & {$[4.0,5.0]$} & {$[1.0,3.0]$} & {$[5.0,6.0]$} & {$[5.0,6.0]$} & {$[3.0,4.0]$} & {$[1.0,3.0]$} \\
\hline $\mathrm{E}_{3}$ & {$[4.0,5.0]$} & {$[1.0,3.0]$} & {$[6.0,9.0]$} & {$[4.0,5.0]$} & {$[4.0,5.0]$} & {$[4.0,5.0]$} & {$[5.0,6.0]$} & {$[3.0,4.0]$} \\
\hline $\mathrm{E}_{4}$ & {$[1.0,3.0]$} & {$[1.0,3.0]$} & {$[5.0,6.0]$} & {$[3.0,4.0]$} & {$[6.0,9.0]$} & {$[5.0,6.0]$} & {$[4.0,5.0]$} & {$[0.0,1.0]$} \\
\hline \multicolumn{9}{|l|}{$A_{3}$} \\
\hline$E_{1}$ & {$[3.0,4.0]$} & {$[4.0,5.0]$} & {$[4.0,5.0]$} & {$[6.0,9.0]$} & {$[6.0,9.0]$} & {$[6.0,9.0]$} & {$[5.0,6.0]$} & {$[3.0,4.0]$} \\
\hline$E_{2}$ & {$[1.0,3.0]$} & {$[4.0,5.0]$} & {$[3.0,4.0]$} & {$[4.0,5.0]$} & {$[9.0,10]$} & {$[5.0,6.0]$} & {$[5.0,6.0]$} & {$[3.0,4.0]$} \\
\hline$E_{3}$ & {$[4.0,5.0]$} & {$[5.0,6.0]$} & {$[5.0,6.0]$} & {$[9.0,10]$} & {$[4.0,5.0]$} & {$[6.0,9.0]$} & {$[5.0,6.0]$} & {$[3.0,4.0]$} \\
\hline $\mathrm{E}_{4}$ & {$[3.0,4.0]$} & {$[3.0,4.0]$} & {$[4.0,5.0]$} & {$[9.0,10]$} & {$[9.0,10]$} & {$[9.0,10]$} & {$[5.0,6.0]$} & {$[3.0,4.0]$} \\
\hline \multicolumn{9}{|l|}{$\mathrm{A}_{4}$} \\
\hline $\mathrm{E}_{1}$ & {$[1.0,3.0]$} & {$[5.0,6.0]$} & {$[6.0,9.0]$} & {$[5.0,6.0]$} & {$[4.0,5.0]$} & {$[4.0,5.0]$} & {$[4.0,5.0]$} & {$[1.0,3.0]$} \\
\hline $\mathrm{E}_{2}$ & {$[0.0,1.0]$} & {$[4.0,5.0]$} & {$[4.0,5.0]$} & {$[4.0,5.0]$} & {$[4.0,5.0]$} & {$[4.0,5.0]$} & {$[3.0,4.0]$} & {$[0.0,1.0]$} \\
\hline$E_{3}$ & {$[3.0,4.0]$} & {$[6.0,9.0]$} & {$[9.0,10]$} & {$[6.0,9.0]$} & {$[5.0,6.0]$} & {$[4.0,5.0]$} & {$[4.0,5.0]$} & {$[3.0,4.0]$} \\
\hline $\mathrm{E}_{4}$ & {$[1.0,3.0]$} & {$[5.0,6.0]$} & {$[9.0,10]$} & {$[5.0,6.0]$} & {$[3.0,4.0]$} & {$[4.0,5.0]$} & {$[5.0,6.0]$} & {$[1.0,3.0]$} \\
\hline
\end{tabular}

Using formula (2), we aggregated grey assessments of the particular sub-processes, and obtained input data to construct the grey decision-making matrix as per formula (3).

Then, using formula (4), we built the normalized grey decision-making matrix (Table 3 ), assuming that all the decision-making criteria we had adopted were profitable in nature (the higher the value, the better). 
Table 3. Values from the normalized grey decision-making matrix.

\begin{tabular}{|c|c|c|c|c|}
\hline & \multicolumn{4}{|c|}{ Normalized grey assessments } \\
\hline & $\mathrm{C}_{1}$ & $\mathrm{C}_{2}$ & $\mathrm{C}_{3}$ & $\mathrm{C}_{4}$ \\
\hline$A_{1}$ & {$[0.31,0.69]$} & {$[0.77,1.00]$} & {$[0.47,0.59]$} & {$[0.47,0.59]$} \\
\hline $\mathrm{A}_{2}$ & {$[0.69,1.00]$} & {$[0.15,0.46]$} & {$[0.59,0.76]$} & {$[0.32,0.47]$} \\
\hline $\mathrm{A}_{3}$ & {$[0.69,1.00]$} & {$[0.62,0.77]$} & {$[0.47,0.59]$} & {$[0.82,1.00]$} \\
\hline $\mathrm{A}_{4}$ & {$[0.31,0.69]$} & {$[0.77,1.00]$} & {$[0.82,1.00]$} & {$[0.59,0.76]$} \\
\hline & $\mathrm{C}_{5}$ & $\mathrm{C}_{6}$ & $\mathrm{C}_{7}$ & $\mathrm{C}_{8}$ \\
\hline $\mathrm{A}_{1}$ & {$[0.32,0.47]$} & {$[0.32,0.47]$} & {$[0.46,0.67]$} & {$[0.75,1.00]$} \\
\hline $\mathrm{A}_{2}$ & {$[0.59,0.76]$} & {$[0.56,0.68]$} & {$[0.67,0.83]$} & {$[0.44,0.75]$} \\
\hline $\mathrm{A}_{3}$ & {$[0.82,1.00]$} & {$[0.76,1.00]$} & {$[0.83,1.00]$} & {$[0.75,1.00]$} \\
\hline $\mathrm{A}_{4}$ & {$[0.47,0.59]$} & {$[0.47,0.59]$} & {$[0.67,0.83]$} & {$[0.31,0.69]$} \\
\hline
\end{tabular}

Based upon data from the normalized grey decision-making matrix and the calculated weights of the particular criteria, we built the weighted normalized decision-making matrix using formula (5) - Table 4.

Table 4. Values from the weighted normalized grey decision-making matrix.

\begin{tabular}{|c|c|c|c|c|}
\hline & \multicolumn{4}{|c|}{ Weighted normalized grey assessments } \\
\hline & $\mathrm{C}_{1}$ & $\mathrm{C}_{2}$ & $\mathrm{C}_{3}$ & $\mathrm{C}_{4}$ \\
\hline$A_{1}$ & {$[0.23,0.61]$} & {$[0.42,0.75]$} & {$[0.21,0.32]$} & {$[0.16,0.26]$} \\
\hline $\mathrm{A}_{2}$ & {$[0.50,0.88]$} & {$[0.08,0.35]$} & {$[0.26,0.42]$} & {$[0.11,0.21]$} \\
\hline $\mathrm{A}_{3}$ & {$[0.50,0.88]$} & {$[0.34,0.58]$} & {$[0.21,0.32]$} & {$[0.29,0.45]$} \\
\hline $\mathrm{A}_{4}$ & {$[0.23,0.61]$} & {$[0.42,0.75]$} & {$[0.37,0.55]$} & {$[0.21,0.34]$} \\
\hline & $\mathrm{C}_{5}$ & $\mathrm{C}_{6}$ & $\mathrm{C}_{7}$ & $\mathrm{C}_{8}$ \\
\hline $\mathrm{A}_{1}$ & {$[0.22,0.43]$} & {$[0.15,0.26]$} & {$[0.17,0.30]$} & {$[0.32,0.58]$} \\
\hline $\mathrm{A}_{2}$ & {$[0.40,0.70]$} & {$[0.25,0.37]$} & {$[0.25,0.38]$} & {$[0.19,0.44]$} \\
\hline $\mathrm{A}_{3}$ & {$[0.56,0.92]$} & {$[0.34,0.55]$} & {$[0.32,0.45]$} & {$[0.32,0.58]$} \\
\hline $\mathrm{A}_{4}$ & {$[0.32,0.54]$} & {$[0.21,0.32]$} & {$[0.25,0.38]$} & {$[0.13,0.40]$} \\
\hline
\end{tabular}

Data from the weighted normalized decision-making matrix was used to identify the pattern as per formula (6), and the anti-pattern as per formula (7). The ideal solution was: $\mathrm{A}^{\max }=\{[0.50,0.88][0.42,0.75][0.42,0.75][0.29,0.45][0.56,0.92][0.34,0.55][0.32,0.45]$ $[0.32,0.58]\}$, whereas the anti-ideal solution was: $\mathrm{A}^{\min }=\{[0.23,0.61][0.08,0.35]$ $[0.21,0.32][0.11,0.21][0.22,0.43][0.15,0.26][0.17,0.30][0.13,0.40]\}$.

Having identified the ideal solution (pattern) and the anti-ideal one (anti-pattern), we calculated distances of the particular alternatives $A_{1}-A_{4}$ using formulas (9) and (10). Calculated distances $\mathrm{d}+$ and $\mathrm{d}-$ are listed in Table 5.

Table 5. List of distances of the alternatives A from the pattern and the anti-pattern.

\begin{tabular}{|r|l|l|l|l|l|l|l|l|}
\hline & $\mathrm{C}_{1}$ & $\mathrm{C}_{2}$ & $\mathrm{C}_{3}$ & $\mathrm{C}_{4}$ & $\mathrm{C}_{5}$ & $\mathrm{C}_{6}$ & $\mathrm{C}_{7}$ & $\mathrm{C}_{8}$ \\
\hline $\mathrm{d}+$ & \multicolumn{7}{|l|}{$\mid$} \\
\hline $\mathrm{A}_{1}$ & 0.27 & 0.00 & 0.20 & 0.16 & 0.42 & 0.25 & 0.15 & 0.00 \\
\hline $\mathrm{A}_{2}$ & 0.00 & 0.37 & 0.12 & 0.21 & 0.19 & 0.14 & 0.07 & 0.14 \\
\hline $\mathrm{A}_{3}$ & 0.00 & 0.13 & 0.20 & 0.00 & 0.00 & 0.00 & 0.00 & 0.00 \\
\hline $\mathrm{A}_{4}$ & 0.27 & 0.00 & 0.00 & 0.10 & 0.32 & 0.19 & 0.07 & 0.19 \\
\hline $\mathrm{d}-$ & \multicolumn{10}{|l|}{} \\
\hline $\mathrm{A}_{1}$ & 0.00 & 0.37 & 0.00 & 0.05 & 0.00 & 0.00 & 0.00 & 0.19 \\
\hline $\mathrm{A}_{2}$ & 0.27 & 0.00 & 0.08 & 0.00 & 0.23 & 0.11 & 0.08 & 0.05 \\
\hline $\mathrm{A}_{3}$ & 0.27 & 0.25 & 0.00 & 0.21 & 0.42 & 0.25 & 0.15 & 0.19 \\
\hline $\mathrm{A}_{4}$ & 0.00 & 0.37 & 0.20 & 0.12 & 0.11 & 0.06 & 0.08 & 0.00 \\
\hline
\end{tabular}


Based upon distances from Table 5, we established synthetic assessment measures of the particular alternatives $C C_{i}$ using formula (11), and then created a ranking of alternatives in descending order: $\operatorname{CC}\left(\mathrm{A}_{3}\right)=0.836 ; \mathrm{CC}\left(\mathrm{A}_{4}\right)=0.451 ; \mathrm{CC}\left(\mathrm{A}_{2}\right)=0.393 ; \mathrm{CC}\left(\mathrm{A}_{1}\right)=0.296$. In line with interpretation of the synthetic assessment measure, it was concluded that bearing in mind the adopted assessment criteria and their particular significance levels, the company that was best in performing its occupational risk management processes was company $A_{3}$, while the one that was the worst in it was company $A_{1}$.

At the same time, a comprehensive view of the occupational risk management process suggests that the key sub-process is the hazard identification sub-process, as any hazard identification that is incorrect, especially in case of critical hazards, affects what this process should address, regardless of whether its successive stages have been correct or not.

Therefore, it is advisable to consider using another approach to identify significance levels of the particular assessment criteria, in such a way as to take into account the impact exerted by the particular criteria upon achievement of the principal objective of the occupational risk management process. Another important problem in the overall discussion is the expert assessments aggregation method we have adopted, which assumed that all the assessment-making experts had the same expertise and competence levels. In this case, it would be advisable to consider possible differentiation of their assessment weights, especially in the situation when experts were not completely independent from the companies they were asked to assess.

\section{Conclusions}

The need to have some tools that offer support in decision-making processes occurs in various management areas in companies, and these tools are used in various areas to a larger or lesser extent $[9,23]$. In this paper, we focus our attention upon the possibility to use a selected decision-making support method in the occupational risk management area. Due to the fact that occupational risk management is an intrinsically complicated system that cannot be described using a single parameter only, the most recommended approach to this process performance assessment would be to use multi-dimensional analysis, and, in consequence, multi-dimensional (multi-criteria) methods. Due to specifics of the particular occupational risk management sub-processes, it is verbal expressions that are most frequently used to assess their performance levels.

Therefore, the objective of our research was to analyze the possibility to use the grey TOPSIS method to assess occupational risk management systems. Although the TOPSIS method, in its classical form, is a ranking method that is perhaps the best known and best described in the literature, the use of grey numbers has broadened its application scope to comprise the work health and safety management system, which the area that has been poorly represented in it so far. It is relatively easy to use the approach discussed in this work. To perform mathematical operations, any spreadsheet can be used, which is especially significant for small and medium-size businesses. Studies and analyses as presented herein confirm that the grey TOPSIS method can be successfully used in the occupational health and safety area to assess, compare, and identify companies that are the best and the worst in performing their occupational risk management processes.

This work may inspire the reader to look for further applications of the grey TOPSIS method, both in this area and in the area of applying grey numbers within the frameworks of other multi-criteria decision-making methods. In the context of the results we have obtained, further research work is planned, covering practical verification of suitability of various methods in solving decision-making problems concerning any remaining crucial work health and safety management issues. 


\section{References}

1. ICE/ISO 31010: 2009

2. J. Tixier, G. Dusserre, O. Salvi, D. Gaston, J. Loss Prevent. Proc. 15, 4 (2002)

3. P. K. Marhavilas, D. Koulouriotis, V. Gemeni, J. Loss Prevent. Proc. 24, 5 (2011)

4. B. W. Main, Prof. Saf. 49, 12 (2012)

5. M. Rausand, Risk Assessment: Theory, Methods, and Applications (John Wiley \& Sons, 2013)

6. V. Holubová, Pol. J. Manage. Stud. 14, 1 (2016)

7. M. Majerník, N. Daneshjo, G. Sančiová, J. Chovancová, Pol. J. Manage. Stud. 15, 1 (2017)

8. ISO 3100: 2009

9. M. Frankovský, Z. Birknerová, L. Zbihlejová, Pol. J. Manage. Stud. 14, 2 (2016)

10. M. Delgado, F. Herrera, E. Herrera-Viedma, L. Martinez, Inform. Sciences 107 (1998)

11. F. Herrera, S. Alonso, F. Chiclana, E. Herrera-Viedma, Fuzzy Optim. Decis. Ma. 8 (2009)

12. Y. J. Xu, Q. I. Da, X. W. Liu, J. Syst. Eng. Electron. 21 (2010)

13. L. Martínez, F. Herrera, Inform. Sciences 207 (2012)

14. ISO 12100: 2010

15. F. H. van Duijne, E. G. Schuoten, D. van Aken, Safety Sci. 46 (2008)

16. J. Moseman, Process Saf. Prog. 31, 1 (2012)

17. F. A. Manuelle, Prof. Saf. 50, 5 (2005)

18. G. H. Tzeng, J. J. Huang, Multiple Attribute Decision Making: Methods and Applications (CRC Press, 2011)

19. C. L. Hwang, K. Yoon, Multiple Attribute Decision Making. Methods and Applications (Springer, Berlin Heidelberg, 1981)

20. S. J. Chen, C. L. Hwang, Fuzzy Multiple Attribute Decision Making: Methods and Applications (Springer, Berlin, 1992)

21. S. Liu, Z. Fang, Y. Yang, J. Forrest, Grey Syst. Theor. Appl. 2, 3 (2012)

22. J. Tabor, Pol. J. Manage. Stud. 18, 1 (2018)

23. R. Rajnoha, J. Dobrovič, Pol. J. Manage. Stud. 15, 2 (2017) 